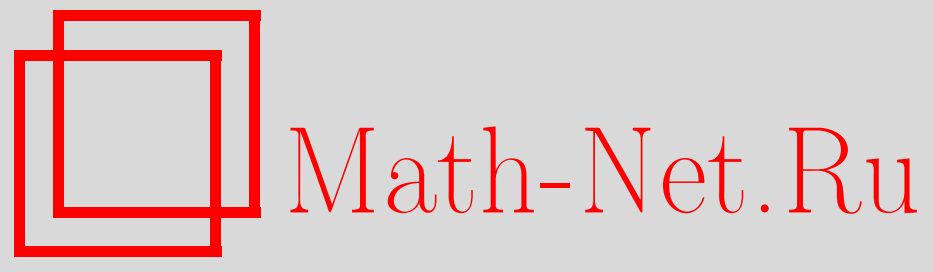

С. Р. Гайнуллова, Т. Н. Фоменко, Функционалы, подчиненные сходящимся рядам, и некоторые приложения, Матем. заметки, 2014, том 96, выпуск 2, 314-317

DOI: https://doi.org/10.4213/mzm10224

Использование Общероссийского математического портала Math-Net.Ru подразумевает, что вы прочитали и согласны с пользовательским соглашением http://www.mathnet.ru/rus/agreement

Параметры загрузки:

IP : 54.198 .67 .100

26 апреля 2023 г., 13:20:25

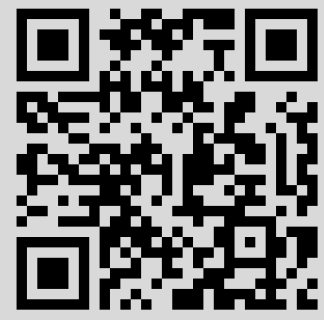




\section{Функционалы, подчиненные сходящимся рядам, и некоторые приложения}

\section{С. Р. Гайнуллова, Т. Н. Фоменко}

В работах [1]-[4] было введено понятие $(\alpha, \beta)$-поискового функционала, рассмотрены его свойства и применения к проблемам существования и дискретного (каскадного) поиска общих неподвижных точек, точек совпадения, общих прообразов заданного замкнутого подпространства, общих корней конечных наборов отображений метрических пространств.

В данном кратком сообщении вводится понятие функционала, подчиненного сходящемуся ряду, являющееся в некотором смысле обобщением понятия $(\alpha, \beta)$-поискового функционала. Рассматриваются применения таких функционалов к решению перечисленных выше задач. Некоторые из представленных результатов анонсированы в [5].

Обозначения и терминология. Далее $\mathbb{R}_{+}=\{t \in \mathbb{R} \mid t \geqslant 0\}$ - множество неотрицательных вещественных чисел; $(X, \rho),(Y, d)$ - метрические пространства; $P(Y)$ - совокупность всевозможных непустых подмножеств пространства $Y ; C(Y)$ - совокупность непустых замкнутых подмножеств пространства $Y ; U(M, r)=\{x \in X \mid \rho(x, M) \leqslant r\}$ - окрестность множества $M$ радиуса $r>0$ в $X, U(x, r)$ - замкнутый шар радиуса $r$ с центром в точке $x$. Метрика $D$ в пространстве $Y^{n}=\underbrace{Y \times \cdots \times Y}_{n}$ задается формулой:

$$
D(y, z):=\sum_{i=1}^{n} d\left(y_{i}, z_{i}\right),
$$

где $y=\left(y_{1}, \ldots, y_{n}\right), z=\left(z_{1}, \ldots, z_{n}\right) \in Y^{n} ; \Delta_{n}=\left\{\widetilde{y}=(y, \ldots, y) \in Y^{n} \mid y \in Y\right\}$ - диагональ в $Y^{n}, n \geqslant 2 ; \Delta_{n}(H)=\left\{\widetilde{y}=(y, \ldots, y) \in \Delta_{n} \mid y \in H\right\}$ - часть диагонали "над $H$ ", где $H$ замкнутое подпространство в $Y$. В частности, $\Delta_{n}=\Delta_{n}(Y)$.

Пусть $f_{1}, \ldots, f_{n}: X \rightarrow Y$ - отображения метрических пространств $(X, \rho),(Y, d)$,

$$
f=f_{1} \times \cdots \times f_{n}: X \rightarrow Y^{n} .
$$

Множество $f^{-1}\left(\Delta_{n}(H)\right)=\left\{x \in X \mid f_{1}(x)=\cdots=f_{n}(x) \in H\right\}$ будем называть множеством общих прообразов подпространства $H$ при действии $f_{1}, \ldots, f_{n}$.

Будем говорить, что график $\operatorname{Gr}(f)=\{(x, y) \in X \times Y \mid y \in f(x)\}$ отображения $f: X \rightarrow Y$ является $H$-полным, если всякая фундаментальная последовательность

$$
\left\{\left(x_{m}, y_{m}\right)\right\}_{m=0,1, \ldots} \subseteq \operatorname{Gr}(f),
$$

где $d\left(y_{m}, H\right) \underset{m \rightarrow \infty}{\longrightarrow} 0$, имеет предел $(\xi, \eta) \in \operatorname{Gr}(f)$. График $\operatorname{Gr}(f)$ называется $H$-замкнутым, если все его предельные точки вида $(x, y)$, где $y \in H$, содержатся в $\operatorname{Gr}(f)$.

Пусть задана неубывающая функция $\mu:[0 ;+\infty) \rightarrow[0 ;+\infty), \mu(0)=0$. Отображение $f$ называется $\mu(t)$-накрывающим в $X$, если $U(f(x), \mu(r)) \subseteq f(U(x, r))$ для всех $x \in X, r>0$. Отображение $g: X \rightarrow Y$ называется $\mu(t)$-сжимающим, если $d\left(g(x), g\left(x^{\prime}\right)\right) \leqslant \mu\left(\rho\left(x, x^{\prime}\right)\right)$ для всех $x, x^{\prime} \in X$.

Мультикаскадом на $X$ называется многозначная дискретная динамическая система с фазовым пространством $X$ и аддитивной полугруппой сдвигов $\left(\mathbb{Z}_{+},+\right), \mathbb{Z}_{+}=\{0,1,2, \ldots\}$, представленной неотрицательными итерациями некоторого (вообще говоря, многозначного) отображения $\mathcal{G}: X \rightarrow P(X)$, называемого генератором мультикаскада. Траекторией мультикаскада называется всякая последовательность $\left\{x_{n}\right\}_{n=1,2, \ldots}$, где $x_{n+1} \in \mathcal{G}\left(x_{n}\right), n=$ $1,2, \ldots$. Пределы траекторий (если они существуют) составляют предельное множество этого мультикаскада.

DOI: $10.4213 / \mathrm{mzm} 10224$ 
Неотрицательный функционал $\varphi: X \rightarrow \mathbb{R}_{+}$называется $(\alpha, \beta)$-поисковым на $X, 0<\beta<\alpha$, если для любого $x \in X$ существует $x^{\prime} \in X$, для которого

$$
\rho\left(x, x^{\prime}\right) \leqslant \frac{\varphi(x)}{\alpha}, \quad \varphi\left(x^{\prime}\right) \leqslant \frac{\beta}{\alpha} \cdot \varphi(x) .
$$

Пусть на $X$ заданы функционал $\varphi: X \rightarrow \mathbb{R}_{+}$и сходящийся ряд

$$
\sum_{n=1}^{+\infty} a_{n}=A, \quad 0<a_{n+1}<a_{n}, \quad n \geqslant 1 .
$$

ОПРеДЕлЕниЕ. Будем говорить, что функционал $\varphi$ подчинен ряду (1), если для любой точки $x \in X$, где $\varphi(x)>0$, существует такая точка $x^{\prime} \in X$, что $\rho\left(x, x^{\prime}\right) \leqslant \varphi(x)$, и $I\left(x^{\prime}\right)>I(x)$, где индекс $I(x)$ точки $x$ относительно пары (функиионал $\varphi$, рлд $(1))$ определен равенством

$$
I(x):= \begin{cases}0, & \varphi(x)>a_{1}, \\ \max \left\{k \in \mathbb{N} \mid \varphi(x) \leqslant a_{k}\right\}, & \text { существует } k \in \mathbb{N} \text { такой, что } 0<\varphi(x) \leqslant a_{k}, \\ \infty, & \varphi(x)=0 .\end{cases}
$$

Простейший пример такого функционала - непрерывная кусочно-линейная функция

$$
\varphi: \mathbb{R}_{+} \rightarrow \mathbb{R}_{+},
$$

где $\varphi(0)=a_{1}, \varphi\left(\sum_{j=1}^{n-1} a_{j}\right)=a_{n}, n \geqslant 2$, линейная на отрезках $\left[0 ; a_{1}\right],\left\{\left[\sum_{j=1}^{n-1} a_{j} ; \sum_{j=1}^{n} a_{j}\right]\right\}_{n \geqslant 2}$, и $\varphi(x)=0$ при $x \geqslant A$.

Несложно проверить, что если $\lim _{n \rightarrow \infty}\left(a_{n+1} / a_{n}\right)=1$, то построенный выше функционал $\varphi(x)$ не является поисковым ни для каких $(\alpha, \beta), 0<\beta<\alpha$. С другой стороны, всякий ограниченный $(\alpha, \beta)$-поисковый функционал $\varphi(x)(0<\beta<\alpha$, существует $C>0$ такое, что $\varphi(x) \leqslant C, x \in X)$ на $(X, \rho)$ подчинен ряду $C \cdot \sum_{k=0}^{\infty}(\beta / \alpha)^{k}$ на пространстве $(X, \alpha \rho)$ с метрикой $\alpha \rho$, эквивалентной $\rho$.

В этом смысле класс функционалов, подчиненных сходящимся рядам вида (1), существенно шире класса $(\alpha, \beta)$-поисковых функционалов.

Отметим, что аналогично можно определить подчиненность функционала сходящемуся функциональному ряду. В данном сообщении мы ограничимся понятием подчиненности функционала числовому ряду.

Теорема 1. Пусть функиионал $\varphi: X \rightarrow \mathbb{R}_{+}$подчинен ряду (1) и выполнено одно из следующих условий:

1) график $\operatorname{Gr}(\varphi)$ является $\{0\}$-полным;

2) пространство $X$ полно и график $\operatorname{Gr}(\varphi)$ является $\{0\}$-замкнутым.

Тогда на $X$ определен мультикаскад, все траектории которого сходятся, и его предельное множество равно $\operatorname{Nil}(\varphi):=\{x \in X \mid \varphi(x)=0\}$. Для любого $x_{0} \in X$, где $\varphi\left(x_{0}\right)>0$, и любой траектории $\left\{x_{j}\right\}_{j=0,1, \ldots}$, выходящей из $x_{0}$ и сходящейся $\kappa \xi \in \operatorname{Nil}(\varphi)$, верно, что

$$
\rho\left(x_{0}, \xi\right) \leqslant \sum_{j=0}^{\infty} a_{I\left(x_{0}\right)+j} .
$$

СХема ДОКАЗАТЕЛЬСТвА. Для любой точки $x_{0} \in X$, где $\varphi\left(x_{0}\right)>0$, условие подчиненности функционала $\varphi$ ряду (1) позволяет построить фундаментальную последовательность $\left\{\left(x_{m}, \varphi\left(x_{m}\right)\right)\right\}_{m=0,1, \ldots}$, которая в силу условий 1$)$ или 2$)$ сходится к некоторой паре $(\xi, 0) \in$ $\operatorname{Gr}(\varphi)$, т.е. $\xi \in \operatorname{Nil}(\varphi)$. Оценка расстояния $\rho\left(x_{0}, \xi\right)$ получается стандартно.

Теорема 1 обобщает результат [1; теорема 1.3], но с другой оценкой $\rho\left(x_{0}, \xi\right)$. 
Теорема 2. Пусть $f_{1}, \ldots, f_{n}: X \rightarrow Y, f=f_{1} \times \cdots \times f_{n}: X \rightarrow Y^{n}$. Пусть $f_{1}$ переводит всякую фундаментальную последовательность в фундаментальную, функиионал $\varphi(x):=$ $D\left(f(x), \Delta_{n}(H)\right)$ подчинен ряду (1) и выполнено одно из условий:

1) график $\operatorname{Gr}(f)$ является $\Delta_{n}(H)$-полным;

2) график $\operatorname{Gr}\left(f_{1}\right)$ H-полон, и график $\operatorname{Gr}(f) \Delta_{n}(H)$-замкнут.

Тогда на $X$ определен мультикаскад с непустым предельным множеством $f^{-1}\left(\Delta_{n}(H)\right)$, все траектории которого сходятся, и для любого $x_{0} \in X$, где $\varphi\left(x_{0}\right)>0$, и любой траектории $\left\{x_{j}\right\}_{j=0,1, \ldots}$, выходящей из $x_{0}$ и сходящейся $\kappa \xi \in f^{-1}\left(\Delta_{n}(H)\right)$, верно, что

$$
\rho\left(x_{0}, \xi\right) \leqslant \sum_{j=0}^{\infty} a_{I\left(x_{j}\right)} \leqslant \sum_{j=0}^{\infty} a_{I\left(x_{0}\right)+j} .
$$

СХемА ДОКАЗАТЕЛЬСТВА. Можно показать, что любое из условий теоремы 1) или 2) обеспечивает $\{0\}$-полноту графика функционала $\varphi(x):=D\left(f(x), \Delta_{n}(H)\right)$. Поэтому утверждение следует из теоремы 1.

Теорема 2 является обобщением [1; теорема 1.7], однако с другой оценкой расстояния $\rho\left(x_{0}, \xi\right)$. В качестве следствий из теоремы 2 получаются утверждения о существовании и аппроксимации совпадений заданного набора отображений (при $H=Y$ ), а также общих корней, соответствующих общему значению $c$ заданного набора отображений (при $H=$ $\{c\})$.

Пусть функции $\mu, \tau:[0 ;+\infty) \rightarrow[0 ;+\infty)$ не убывают, $\mu(0)=\tau(0)=0, \mu(t)$ обратима и непрерывна в нуле справа. Пусть также $\left(\mu^{-1} \circ \tau\right)(t) \leqslant a_{n}$ при $a_{n}<t \leqslant a_{n-1}, n \geqslant 2$, и $\left(\mu^{-1} \circ \tau\right)(t) \leqslant a_{1}$ при $t>a_{1}$.

Теорема 3 (Т. Н. Фоменко). Пусть $f, g: X \rightarrow Y$. Пусть отображение $f$ является $\mu(t)$ накрывающим, а отображение $g-\tau(t)$-сжимающим. Пусть также одно из отображений $f, g$ переводит всякую фундаментальную последовательность в фундаментальную, и его график полон, а график второго отображения замкнут. Тогда на X существует мультикаскад с предельным множеством

$$
\operatorname{Coin}(f, g)=\{x \in X \mid f(x)=g(x)\} \neq \varnothing,
$$

все траектории которого сходятся. Если $\mu^{-1}\left(d\left(f\left(x_{0}\right), g\left(x_{0}\right)\right)\right) \leqslant a_{k_{0}}$ для некоторых $x_{0} \in X$, $k_{0} \in \mathbb{N}$, то для любой траектории $\left\{x_{m}\right\}_{m=0,1, \ldots}$, начинающейся из $x_{0}$ и сходящейся $\kappa \xi \in$ $\operatorname{Coin}(f, g)$, верно

$$
\rho\left(x_{0}, \xi\right) \leqslant \sum_{j=0}^{\infty} a_{k_{0}+j} .
$$

СХемА ДоКАЗАТЕЛЬСТвА. Условия теоремы обеспечивают подчиненность функционала $\varphi(x):=\mu^{-1}(d(f(x), g(x))), x \in X$, ряду (1) и $\{0\}$-полноту его графика. Поэтому утверждение следует из теоремы 1.

Отметим, что теорема 3 представляет обобщение [6; теорема 1], но с другой оценкой на расстояние $\rho\left(x_{0}, \xi\right)$.

Если отображение $f$ тождественно, а отображение $g$ является $\tau(t)$-сжимающим, то из теоремы 3 получается утверждение, близкое к результату [7; лемма 2], для случая однозначных отображений.

Отметим, что применение функционалов, подчиненных сходящимся рядам, к задаче о совпадениях семейства пар отображений, параметризованных элементами некоторого топологического пространства, содержится в статье [8]. Близкое понятие функционалов, строго подчиненных сходящимся рядам, и их применение к решению некоторых уравнений в метрических пространствах дано в [9].

Авторы искренне благодарят рецензента за ряд полезных замечаний и предложений по улучшению изложения. 


\section{СПИСОК ЦИТИРОВАННОЙ ЛИТЕРАТУРЫ}

[1] T. N. Fomenko, Topology Appl., 157:4 (2010), 760-773. [2] Т. Н. Фоменко, Изв. РАН. Сер. матем., 74:5 (2010), 171-190. [3] Т. Н. Фоменко, Вестн. Моск. ун-та. Сер. 1. Матем., мех., 2010, № 5, 3-9. [4] Т. Н. Фоменко, Матем. заметки, 93:1 (2013), 127-143. [5] С. Р. Гайнуллова, "Каскадный поиск нулей функционала, подчиненного сходящемуся ряду", ХІХ Международная научная конференция студентов, аспирантов и молодых ученых “Ломоносов-2012", Сб. тезисов, М., 2012, 74-75. [6] А.В. Арутюнов, Докл. РАН, 416:2 (2007), 151-155. [7] П. В. Семенов, Функи. анализ и его прил., 36:2 (2002), 89-92. [8] C. P. Гайнуллова, Int. J. Open Inform. Tech., 2:5 (2014), 10-16. [9] T. N. Fomenko, J. Fixed Point Theory Appl., 14:1 (2013), 21-40.

\section{С. Р. Гайнуллова}

Московский государственный университет имени М. В. Ломоносова

E-mail: gajnullova-sr@yandex.ru

\section{Т. Н. Фоменко}

Московский государственный университет имени М. В. Ломоносова

E-mail: tn-fomenko@yandex.ru
Поступило

14.09.2012

Исправленный вариант

11.11.2013 